\title{
Optimizing Patient care through Effective Pharmacy Practice Models
}

\author{
Madeeha Malik, Azhar Hussain \\ Hamdard Institute of Pharmaceutical Sciences, Hamdard University, Pakistan
}

Rational use of medicines is very much reliant on the healthcare systems within which care is delivered. There has been a dialogue for quite some time on potential of pharmacy practice for optimizing patient care globally. The contributions of pharmacy practice has helped many countries in achieving better health outcomes, reducing the costs of treatment, building the patient trust on healthcare system, reduced side effects and risk of treatments, decreased morbidity and mortality, improved access to safe and timely treatments and improved quality of life resulting in achievements of millennium development goals. Countries which have adopted improved pharmacy practice models have endowed enormous resources in their healthcare systems. However, it is not feasible to pertain a single change model to pharmacy service provision worldwide, due to disparity among healthcare systems of different countries. Thus, the major challenges and their solutions in designing optimizing patient care models along with in-depth mechanism related to the strategies for overcoming irrational use of drugs needs to be explicitly investigated.

This special issue of American Journal of Pharmacological Sciences brings together a collection of insightful papers that address some of these issues related to various strategies which can help to promote rational use of drugs. The goal of this special issue is to highlight different strategies which can improve patient care and to let the readers become more knowledgeable regarding recent progression in optimizing patient care through effective pharmacy practice models. The first paper has underlined the need for an action plan for all stakeholders to understand their role and commit to shared goals for achieving better healthcare outcomes. The paper highlighted that inclusion of status of pharmacist as provider care, implementation of integrated hospital and community practice models, capacity building, training and research by involving all stakeholders is the ultimate action plan for implementation of effective pharmacy practice model. The second review paper has evaluated the cost effectiveness of a feign one-year vaccination intervention using the oral polio vaccine (OPV) in Kano State, Nigeria, in global polio eradication fight for developing nations. The author reported cost effectiveness of the intervention which implies that vaccination program is economically viable and should therefore be encouraged, than just concentrating on treating polio. The author proposed that with the economic crunch times in the world, there is a strong urge for cost effective programs to address the deadly diseases and the evaluated effective program would be one of them. The next paper evaluated the efficacy of local and multinational brands of ciprofloxacin used in treatment of typhoid. Results showed that local brands of ciprofloxacin were relatively better in terms of bioavailability, efficacy and cost. Availability of brands having sub-standard levels of the active agent results in resistance to typhoid and ultimately increases the cost of therapy. On the other hand, local brands can be used as therapeutic alternatives to multinational brands which showed greater activity against microorganism and had maximum drug content with least price in market. So Government and Ministry of health should take necessary steps in promoting cost effective treatment of typhoid, in regulating quality and standard of allopathic formulations. Thromboprophylaxis is the mainstay therapy for preventing thromboembolic events such as stroke in Atrial Fibrillation patients. Patients with moderate or high risk of stroke should receive warfarin as it has shown to be superior in clinical trials, with increase in relative reduction of stroke and similar incidence of bleeding compared to aspirin alone. The next paper identified the common types and frequencies of pharmaceutical care issues in AF patients receiving thromboprohylaxis and the use of thromboprophylactic agents in accordance to stroke risk stratification. The paper reported identification of varieties of PCIs in AF patients receiving thromboprophylaxis which support the need of dedicated clinical pharmacist to work in collaboration with healthcare team and patients. Health related quality of life (HRQoL) is used to measure both the present health status and the impact of health care interventions on the patients. The last paper evaluated improvement in health related quality of life of hypertensive patients after pharmaceutical care provided by pharmacist. The paper reported that health related quality of life of hypertensive patients were significantly improved after pharmaceutical care intervention. 